Mathematical Modelling and Analysis

Volume 21 Number 3, May 2016, 304-318

http://dx.doi.org/10.3846/13926292.2016.1155507

(c) Vilnius Gediminas Technical University, 2016
Publisher: Taylor\&Francis and VGTU

http://www.tandfonline.com/TMMA

ISSN: $1392-6292$

eISSN: $1648-3510$

\title{
On Solutions of Boundary Value Problem for Fourth-Order Beam Equations
}

\section{Lazhar Bougoffa ${ }^{a}$, Randolph Rach $^{b}$ and Abdul-Majid Wazwaz ${ }^{c}$}

\author{
${ }^{a}$ Al Imam Mohammad Ibn Saud Islamic University (IMSIU) \\ Faculty of Science, Department of Mathematics, \\ P.O. Box 90950, 11623 Riyadh, Saudi Arabia \\ $b$ The George Adomian Center for Applied Mathematics \\ 316 South Maple Street, 49057-1225 Hartford, MI, USA \\ ${ }^{c}$ Department of Mathematics, Saint Xavier University \\ Chicago, 60655 IL, USA \\ E-mail(corresp.): bougoffa@hotmail.com \\ E-mail: tapstrike@gmail.com \\ E-mail: wazwaz@sxu.edu
}

Received September 17, 2015; revised February 11, 2016; published online May 15, 2016

\begin{abstract}
In this paper, we consider the fourth-order linear differential equation $u^{(4)}+f(x) u=g(x)$ subject to the mixed boundary conditions $u(0)=u(1)=u^{\prime \prime}(0)=$ $u^{\prime \prime}(1)=0$. We first establish sufficient conditions on $f(x)$ that guarantee a unique solution of this problem in the Hilbert space by using an a priori estimate. Accurate analytic solutions in series forms are obtained by a new variation of the Duan-Rach modified Adomian decomposition method (DRMA), and then extend this approach to some boundary value problems of fourth-order nonlinear beam equations. Also, a comparison of the two approximate solutions by the ADM with the Green function approach is presented.
\end{abstract}

Keywords: fourth-order equation, a priori estimate, Duan-Rach modified Adomian decomposition method, Adomian polynomials.

AMS Subject Classification: 34B05; 34B10; 34B18.

\section{Introduction}

Consider the real-valued two-point fourth-order linear boundary problem $[6$, $17,20]$

$$
\begin{aligned}
& u^{(4)}(x)+f(x) u(x)=g(x), 0<x<1, \\
& u(0)=0, u(1)=0, u^{\prime \prime}(0)=0, u^{\prime \prime}(1)=0,
\end{aligned}
$$


where $f$ and $g$ are continuous functions on $[0,1]$. This problem naturally arises in various areas of physics, engineering and mathematics such as the plate deflection theory. The analytical solution of problem (1.1)-(1.2) is given by Timoshenko and Woinowsky-Krieger [16] provided that the functions $f(x)$ and $g(x)$ are constants.

In [17], the author established a sufficient condition inf $f(x)=-\alpha>-\pi^{4}$, that is $-f(x)<\alpha$, that guarantees a unique solution for problem (1.1)-(1.2). Also, the existence and uniqueness theorem for the positivity (or negativity) of solutions is given in [6].

In the present work, we study the above problem with sufficient conditions on $f(x)$ that guarantee a unique solution in the Hilbert space. Also, accurate analytic solutions in series form for fourth-order linear and some nonlinear beam equations are obtained by a new variation of the Duan-Rach modified Adomian decomposition method (DRMA) [7,8,9,10].

For the sake of comparison, we will solve two of the proposed examples by the standard Adomian decomposition method $[1,2,3,5,9,18,19]$ and $[7,8,9,10]$.

\section{The uniqueness theorem}

The purpose of this section is to establish sufficient conditions on $f(x)$ that guarantee a unique solution for a linear operator equation of the form

$$
\digamma u=g, u \in U
$$

where $\digamma: D(\digamma) \subset U \rightarrow V$,

$$
\digamma u=u^{(4)}+f(x) u
$$

and where $U$ is a Hilbert space:

$$
U=\left\{u: u, \frac{d^{i} u}{d x^{i}} \in L_{2}(0,1), i=1,2,3,4\right\}
$$

with respect to the norm

$$
\begin{gathered}
\|u\|_{U}^{2}=\int_{0}^{1}\left[u^{2}+\left(\frac{d u}{d x}\right)^{2}+\left(\frac{d^{2} u}{d x^{2}}\right)^{2}+\left(\frac{d^{3} u}{d x^{3}}\right)^{2}+\left(\frac{d^{4} u}{d x^{4}}\right)^{2}\right] d x<\infty, \\
D(\digamma)=\left\{u \in U, u(0)=u(1)=0 \text { and } u^{\prime \prime}(0)=u^{\prime \prime}(1)=0\right\}
\end{gathered}
$$

and $V=L_{2}(0,1)$ is a Hilbert space with respect to the norm

$$
\|g\|_{L_{2}}^{2}=\int_{0}^{1} g^{2}(x) d x .
$$

For $u \in U$, we define the operator $M$ by

$$
M u \equiv u-\frac{d^{2} u}{d x^{2}}
$$


Firstly, consider the scalar product $(\digamma u, u)_{L_{2}}$. Employing integration by parts, and taking into account that $u(0)=u(1)=0$ and $u^{\prime \prime}(0)=u^{\prime \prime}(1)=0$, we obtain

$$
(\digamma u, u)_{L_{2}}=\int_{0}^{1}\left(\frac{d^{2} u}{d x^{2}}\right)^{2} d x+\int_{0}^{1} f(x) u^{2} d x
$$

Now, consider $\left(\digamma u,-\frac{d^{2} u}{d x^{2}}\right)_{L_{2}}$. Employing integration by parts, and taking into account the aforementioned boundary conditions, we obtain

$$
\left(\digamma u,-\frac{d^{2} u}{d x^{2}}\right)_{L_{2}}=\int_{0}^{1}\left(\frac{d^{3} u}{d x^{3}}\right)^{2} d x+\int_{0}^{1} f(x)\left(\frac{d u}{d x}\right)^{2} d x-\frac{1}{2} \int_{0}^{1} f^{\prime \prime}(x) u^{2} d x .
$$

Adding (2.1) and (2.2), we obtain

$$
\begin{aligned}
(\digamma u, M u)_{L_{2}} & =\int_{0}^{1}\left(\frac{d^{2} u}{d x^{2}}\right)^{2} d x+\int_{0}^{1} f(x) u^{2} d x+\int_{0}^{1}\left(\frac{d^{3} u}{d x^{3}}\right)^{2} d x \\
& +\int_{0}^{1} f(x)\left(\frac{d u}{d x}\right)^{2} d x-\frac{1}{2} \int_{0}^{1} f^{\prime \prime}(x) u^{2} d x
\end{aligned}
$$

The scalar product $(\digamma u, M u)_{L_{2}}$ can be estimated by means of the CauchySchwarz-Bunyakovski inequality and the $\varepsilon-$ inequality

$$
\begin{aligned}
& 2 u v \leq \varepsilon u^{2}+\frac{1}{\varepsilon} v^{2}, u, v \geq 0, \varepsilon>0 \\
&\left|(\digamma u, M u)_{L_{2}}\right| \leq \frac{1}{2 \varepsilon_{1}} \int_{0}^{1} g^{2}(x) d x+\frac{\varepsilon_{1}}{2} \int_{0}^{1} u^{2}(x) d x \\
& \quad+\frac{1}{2 \varepsilon_{2}} \int_{0}^{1} g^{2}(x) d x+\frac{\varepsilon_{2}}{2} \int_{0}^{1}\left(\frac{d^{2} u}{d x^{2}}\right)^{2} d x
\end{aligned}
$$

If we suppose that $\alpha_{1} \leq f(x) \leq \alpha_{2}$ and $-f^{\prime \prime}(x)>\beta>0$ for all $x \in[0,1]$, then

$$
\begin{aligned}
& \left(\alpha_{1}+\frac{\beta}{2}-\varepsilon_{1}\right) \int_{0}^{1} u^{2} d x+\alpha_{1} \int_{0}^{1}\left(\frac{d u}{d x}\right)^{2} d x+\left(1-\varepsilon_{2}\right) \int_{0}^{1}\left(\frac{d^{2} u}{d x^{2}}\right)^{2} d x \\
& +\int_{0}^{1}\left(\frac{d^{3} u}{d x^{3}}\right)^{2} d x \leq\left(\frac{1}{2 \varepsilon_{1}}+\frac{1}{2 \varepsilon_{2}}\right) \int_{0}^{1} g^{2}(x) d x
\end{aligned}
$$

From equation (1.1), we have

$$
\frac{d^{4} u}{d x^{4}}=g(x)-f(x) u
$$

and therefore it follows that

$$
\varepsilon_{3} \int_{0}^{1}\left(\frac{d^{4} u}{d x^{4}}\right)^{2} d x \leq 2 \varepsilon_{3}\left(\int_{0}^{1} g^{2}(x) d x+\alpha_{2}^{2} \int_{0}^{1} u^{2} d x\right), \varepsilon_{3}>0 .
$$


Adding this estimate with inequality (2.3), we obtain

$$
\begin{aligned}
& \left(\alpha_{1}+\frac{\beta}{2}-\varepsilon_{1}-2 \varepsilon_{3} \alpha_{2}^{2}\right) \int_{0}^{1} u^{2} d x+\alpha_{1} \int_{0}^{1}\left(\frac{d u}{d x}\right)^{2} d x+\left(1-\varepsilon_{2}\right) \int_{0}^{1}\left(\frac{d^{2} u}{d x^{2}}\right)^{2} d x \\
& +\int_{0}^{1}\left(\frac{d^{3} u}{d x^{3}}\right)^{2} d x+\varepsilon_{3} \int_{0}^{1}\left(\frac{d^{4} u}{d x^{4}}\right)^{2} d x \leq\left(2 \varepsilon_{3}+\frac{1}{2 \varepsilon_{1}}+\frac{1}{2 \varepsilon_{2}}\right) \int_{0}^{1} g^{2}(x) d x .
\end{aligned}
$$

Choosing $\varepsilon_{i}, i=1,2,3$ to be sufficiently small so that $\alpha_{1}+\frac{\beta}{2}-\varepsilon_{1}-2 \varepsilon_{3} \alpha_{2}^{2}>0$. Hence

$$
\int_{0}^{1}\left[u^{2}+\left(\frac{d u}{d x}\right)^{2}+\left(\frac{d^{2} u}{d x^{2}}\right)^{2}+\left(\frac{d^{3} u}{d x^{3}}\right)^{2}+\left(\frac{d^{4} u}{d x^{4}}\right)^{2}\right] d x \leq C \int_{0}^{1} g^{2}(x) d x
$$

that is

$$
\|u\|_{U} \leq C_{1}\|g\|_{L_{2}}
$$

where $C_{1}=C^{\frac{1}{2}}$ and $C=\frac{2+1 /\left(2 \varepsilon_{1}\right)+1\left(2 \varepsilon_{2}\right)}{\min \left(\alpha+\beta / 2-\varepsilon_{1}-2 \varepsilon_{3} \alpha_{2}^{2}, \alpha, 1-\varepsilon_{2}, \varepsilon_{3}\right)}$.

Thus we have proved the following statement.

Theorem 1. Suppose that $\alpha_{1} \leq f(x) \leq \alpha_{2}$ and $-f^{\prime \prime}(x)>\beta>0$ for all $x \in[0,1]$. Then for $g \in L_{2}[0,1]$, there exists a constant $C_{1}>0$ such that the obtained a priori estimate (2.4) holds.

Notice that the uniqueness of the solution follows from the stability estimate $(2.4)$.

\section{Approximate analytic solution by the Duan-Rach modified Adomian decomposition method (DRMA)}

We propose here to solve this fourth-order linear boundary value problem by the Duan-Rach modified Adomian decomposition method (DRMA) [7,8,9,10].

\subsection{The linear problem}

Consider equation (1.1) with the general nonhomogeneous mixed boundary conditions

$$
u(0)=\alpha_{1}, u(1)=\alpha_{2}, u^{\prime \prime}(0)=\beta_{1}, u^{\prime \prime}(1)=\beta_{2} .
$$

This problem can be converted into the following system of two-coupled boundary value problems

$$
\left\{\begin{aligned}
u^{\prime \prime} & =v, u(0)=\alpha_{1}, u(1)=\alpha_{2} \\
v^{\prime \prime}+f(x) u & =g(x), v(0)=\beta_{1}, v(1)=\beta_{2}
\end{aligned}\right.
$$

Next we rewrite this system in Adomian's operator-theoretic notation as

$$
\left\{\begin{aligned}
\mathcal{L} u & =v, u(0)=\alpha_{1}, u(1)=\alpha_{2} \\
\mathcal{L} v+R u & =g, v(0)=\beta_{1}, v(1)=\beta_{2}
\end{aligned}\right.
$$


where

$$
\mathcal{L}(\cdot)=\frac{d^{2}}{d x^{2}}(\cdot) \text { and } R u=f(x) u .
$$

Applying the inverse operator

$$
\mathcal{L}^{-1}(\cdot)=\int_{0}^{x} \int_{0}^{y}(\cdot) d t d y
$$

to both sides of the two equations of system (3.2) and taking into account $u(0)=\alpha_{1}$ and $v(0)=\beta_{1}$, we obtain

$$
\left\{\begin{array}{l}
u(x)=\alpha_{1}+A x+\int_{0}^{x} \int_{0}^{y} v(t) d t d y \\
v(x)=\beta_{1}+B x-\int_{0}^{x} \int_{0}^{y} f(t) u(t) d t d y+\int_{0}^{x} \int_{0}^{y} g(t) d t d y,
\end{array}\right.
$$

where $A=u^{\prime}(0)$ and $B=v^{\prime}(0)=u^{\prime \prime \prime}(0)$ are unknowns constants. The values of $A$ and $B$ can be determined by using the remaining boundary conditions $u(1)=\alpha_{2}$ and $u^{\prime \prime}(1)=\beta_{2}$.

Evaluation of $u(x)$ and $v(x)$ at $x=1$ yields

$$
\left\{\begin{array}{l}
u(1)=\alpha_{1}+A+\int_{0}^{1} \int_{0}^{y} v(t) d t d y=\alpha_{2} \\
v(1)=\beta_{1}+B-\int_{0}^{1} \int_{0}^{y} f(t) u(t) d t d y+\int_{0}^{1} \int_{0}^{y} g(t) d t d y=\beta_{2} .
\end{array}\right.
$$

Thus

$$
\left\{\begin{array}{l}
A=\alpha_{2}-\alpha_{1}-\int_{0}^{1} \int_{0}^{y} v(t) d t d y \\
B=\beta_{2}-\beta_{1}+\int_{0}^{1} \int_{0}^{y} f(t) u(t) d t d y-\int_{0}^{1} \int_{0}^{y} g(t) d t d y
\end{array}\right.
$$

Upon substitution of these values into system (3.3), we have

$$
\left\{\begin{aligned}
u(x) & =\alpha_{1}+\left[\alpha_{2}-\alpha_{1}-\int_{0}^{1} \int_{0}^{y} v(t) d t d y\right] x+\int_{0}^{x} \int_{0}^{y} v(t) d t d y \\
v(x) & =\beta_{1}+\left[\beta_{2}-\beta_{1}+\int_{0}^{1} \int_{0}^{y} f(t) u(t) d t d y-\int_{0}^{1} \int_{0}^{y} g(t) d t d y\right] x \\
& -\int_{0}^{x} \int_{0}^{y} f(t) u(t) d t d y+\int_{0}^{x} \int_{0}^{y} g(t) d t d y
\end{aligned}\right.
$$

which is the equivalent linear Fredholm-Volterra integral system without any undetermined constants of integration.

Define the solutions $u(x)$ and $v(x)$ by their respective infinite series of components in the form

$$
u(x)=\sum_{n=0}^{\infty} u_{n}(x) \text { and } v(x)=\sum_{n=0}^{\infty} v_{n}(x)
$$

Thus, the components $u_{n}$ and $v_{n}$ can be elegantly determined by setting the new modified recursion scheme

$$
\left\{\begin{aligned}
u_{0} & =\alpha_{1}+\left(\alpha_{2}-\alpha_{1}\right) x, v_{0}=\beta_{1}+\left(\beta_{2}-\beta_{1}\right) x+\int_{0}^{x} \int_{0}^{y} g(t) d t d y, \\
u_{n+1} & =-\left[\int_{0}^{1} \int_{0}^{y} v_{n}(t) d t d y\right] x+\int_{0}^{x} \int_{0}^{y} v_{n}(t) d t d y, n \geq 0, \\
v_{n+1} & =\left[\int_{0}^{1} \int_{0}^{y} f(t) u_{n}(t) d t d y\right] x-\int_{0}^{x} \int_{0}^{y} f(t) u_{n}(t) d t d y, n \geq 0 .
\end{aligned}\right.
$$




\subsection{The nonlinear problem}

Consider the nonlinear problem [20]

$$
u^{(4)}=f\left(x, u, u^{\prime \prime}\right), 0<x<1,
$$

subject to the set of the nonhomogeneous mixed boundary conditions (3.1).

In [20], the author established the following general result for this problem.

Theorem 2. Suppose that $f(x, y, z)$ is continuous on $[0,1] \times R \times R$ and there are constants $a, b, c>0$ such that

$$
|f(x, y, z)| \leq a|y|+b|z|+c,
$$

where $\frac{a}{\pi^{4}}+\frac{b}{\pi^{2}}<1$. Then problem (3.5), (3.1) has a solution.

Also, a class of this type of boundary value problems can be found in [4], where the authors studied the existence, uniqueness and multiplicity of positive solutions.

In a similar manner, this problem can be converted into the following system

$$
\left\{\begin{array}{l}
u^{\prime \prime}=v, u(0)=\alpha_{1}, u(1)=\alpha_{2}, \\
v^{\prime \prime}=f(x, u, v), v(0)=\beta_{1}, v(1)=\beta_{2}
\end{array}\right.
$$

and therefore in Adomian's operator-theoretic notation as

$$
\left\{\begin{array}{l}
\mathcal{L} u=v, u(0)=\alpha_{1}, u(1)=\alpha_{2}, \\
\mathcal{L} v=N u, v(0)=\beta_{1}, v(1)=\beta_{2},
\end{array}\right.
$$

where $N u=f\left(x, u, u^{\prime \prime}\right)$. The Adomian decomposition method admits the use of the infinite decomposition series

$$
u=\sum_{n=0}^{\infty} u_{n} \text { and } v=\sum_{n=0}^{\infty} v_{n}
$$

for the solutions $u(x)$ and $v(x)$, respectively, and the infinite series

$$
N(u)=\sum_{n=0}^{\infty} A_{n}\left(u_{0}, u_{1}, \ldots, u_{n}\right)
$$

for the nonlinear term $N(u)$, where the $A_{n}$ are the Adomian polynomials $[1,2$, $3,18,19]$, which can be obtained from the definitional formula

$$
A_{n}=\frac{1}{n !} \frac{d^{n}}{d \lambda^{n}}\left[f\left(\sum_{i=0}^{n} \lambda^{i} y_{i}\right)\right]_{\lambda=0}, n=0,1,2, \ldots .
$$

Consequently the components $u_{n}$ and $v_{n}$ of the solution $u(x)$ and $v(x)$, respectively, will be determined recursively by setting the new modified recursion scheme

$$
\left\{\begin{aligned}
u_{0} & =\alpha_{1}+\left(\alpha_{2}-\alpha_{1}\right) x, v_{0}=\beta_{1}+\left(\beta_{2}-\beta_{1}\right) x \\
u_{n+1} & =-\left[\int_{0}^{1} \int_{0}^{y} v_{n}(t) d t d y\right] x+\int_{0}^{x} \int_{0}^{y} v_{n}(t) d t d y, n \geq 0 \\
v_{n+1} & =-\left[\int_{0}^{1} \int_{0}^{y} A_{n}(t) d t d y\right] x+\int_{0}^{x} \int_{0}^{y} A_{n}(t) d t d y, n \geq 0
\end{aligned}\right.
$$




\subsection{A three-point boundary value problem}

Consider the fourth-order beam equation [11]

$$
u^{(4)}=g(x) f(u(x)), 0<x<1,
$$

subject to the boundary conditions

$$
u(0)=\alpha_{1}, u^{\prime}(0)=\alpha_{2}, u^{\prime \prime}(\beta)=\gamma_{1}, u^{\prime \prime}(1)=\gamma_{2},
$$

where $g(x) \neq 0$ on $[0,1]$ and $\beta \in[2 / 3,1)$ is a constant. This type of problems was considered in [11] as a three-point boundary value problem for the beam equation with $\alpha_{i}=\gamma_{i}=0, i=1,2$, where the authors established some a priori estimates to the positive solutions and established sufficient conditions for the existence and nonexistence of positive solutions.

We rewrite this problem as an equivalent system

$$
\left\{\begin{array}{l}
\mathcal{L}_{1} u \equiv u^{\prime \prime}=v, u(0)=\alpha_{1}, u^{\prime}(0)=\alpha_{2}, \\
\mathcal{L}_{2} v \equiv v^{\prime \prime}=g(x) f(u), v(\beta)=\gamma_{1}, v(1)=\gamma_{2}
\end{array}\right.
$$

We can proceed as before with

$$
\mathcal{L}_{1}^{-1}(.)=\int_{0}^{x} \int_{0}^{y}(.) d t d y \text { and } \mathcal{L}_{2}^{-1}(.)=\int_{1}^{x} \int_{0}^{y}(.) d t d y
$$

to obtain

$$
\left\{\begin{array}{l}
u(x)=\alpha_{1}+\alpha_{2} x+\int_{0}^{x} \int_{0}^{y} v(t) d t d y \\
v(x)=\gamma_{2}+A(x-1)+\int_{1}^{x} \int_{0}^{y} g(t) f(u(t)) d t d y
\end{array}\right.
$$

where $A=v^{\prime}(0)=u^{\prime \prime \prime}(0)$ is an unknown constant and can be determined by using the remaining boundary condition $v(\beta)=u^{\prime \prime}(\beta)=\gamma_{1}$. Thus

$$
A=\frac{1}{\beta-1}\left(\gamma_{1}-\gamma_{2}-\int_{1}^{\beta} \int_{0}^{y} g(t) f(u(t)) d t d y\right) .
$$

Consequently, the components $u_{n}$ and $v_{n}$ can be elegantly determined by setting the new modified recursion scheme

$$
\left\{\begin{array}{l}
u_{0}=\alpha_{1}+\alpha_{2} x, v_{0}=\gamma_{2}+\frac{1}{\beta-1}\left(\gamma_{2}-\gamma_{1}\right)+\frac{1}{\beta-1}\left(\gamma_{1}-\gamma_{2}\right) x \\
u_{n+1}=\int_{0}^{x} \int_{0}^{y} v_{n}(t) d t d y, n \geq 0 \\
v_{n+1}=\frac{1}{\beta-1}\left[\int_{1}^{\beta} \int_{0}^{y} g(t) A_{n}(t) d t d y\right](1-x)+\int_{1}^{x} \int_{0}^{y} g(t) A_{n}(t) d t d y, n \geq 0
\end{array}\right.
$$

where the $A_{n}$ are the Adomian polynomials for the nonlinear term $N u=f(u)$.

\subsection{Examples}

Example 1. Consider the following fourth-order boundary value problem

$$
\begin{aligned}
& y^{(4)}+\left(1-\frac{x^{2}}{2}\right) y=-\frac{x^{4}}{2}+\frac{x^{3}}{2}+x^{2}-x, \quad 0<x<1, \\
& y(0)=0, \quad y(1)=0, y^{\prime \prime}(0)=2, \quad y^{\prime \prime}(1)=2 .
\end{aligned}
$$


First of all, we begin our approach by replacing the inhomogeneous boundary conditions by the corresponding homogeneous conditions. To do this, we introduce a new unknown function $u(x)$ such that $y(x)=u(x)+x^{2}-x$. Then $u$ satisfies the following boundary value problem

$$
\begin{aligned}
& u^{(4)}+\left(1-\frac{x^{2}}{2}\right) u=0, \quad 0<x<1, \\
& u(0)=0, u(1)=0, u^{\prime \prime}(0)=0, u^{\prime \prime}(1)=0 .
\end{aligned}
$$

Here $f(x)=1-\frac{x^{2}}{2}$ and $g(x)=0$. A simple calculation leads to $-f^{\prime \prime}(x)=1$. Thus the conditions of Theorem 1 are satisfied. The recursion scheme (3.4) produces a rapidly convergent series as

$$
\left\{\begin{array}{l}
u_{0}=0, v_{0}=0 \\
u_{n}=0, v_{n}=0, n \geq 1
\end{array}\right.
$$

Consequently, the intermediate solution $u(x)=0$. Returning to the original dependent variable, we obtain $y(x)=x^{2}-x$, which is the exact solution to this problem.

Using the standard Adomian decomposition method. For the sake of comparison, we will solve this proposed example by the standard Adomian decomposition method.

In an operator form, we rewrite this equation as

$$
\mathcal{L} y+\left(1-\frac{x^{2}}{2}\right) y=-\frac{x^{4}}{2}+\frac{x^{3}}{2}+x^{2}-x, 0<x<1
$$

with the given boundary conditions, where $\mathcal{L}$ is a fourth-order differential operator, where we assume that the corresponding integral operator $\mathcal{L}^{-1}$ exists. Applying the inverse operator $\mathcal{L}^{-1}$ to both sides of (3.6) gives

$$
y(x)=\alpha x+x^{2}+\frac{\beta x^{3}}{6} x^{3}-\frac{x^{8}}{3360}+\frac{x^{7}}{1680}+\frac{x^{6}}{360}-\frac{5 x^{5}}{120}-\mathcal{L}^{-1}\left(1-\frac{x^{2}}{2}\right) y,
$$

where the unknown constants $\alpha=y^{\prime}(0), \beta=y^{\prime \prime \prime}(0)$ will be determined later by using the other boundary conditions.

The ADM admits the use of the infinite decomposition series

$$
y(x)=\sum_{n=0}^{\infty} y_{n}(x)
$$

This assumption allows us to use the following recursion relation

$$
\begin{aligned}
y_{0}(x) & =f(x), \\
y_{k+1}(x) & =-\mathcal{L}^{-1}\left(1-\frac{x^{2}}{2}\right) y_{k}, k \geq 0,
\end{aligned}
$$


where $f(x)$ is given by all terms that are not included under the inverse operator $\mathcal{L}^{-1}$. This in turn gives the following solution components

$$
\begin{aligned}
& y_{0}(x)=\alpha x+x^{2}+\frac{\beta x^{3}}{6}-\frac{x^{8}}{3360}+\frac{x^{7}}{1680}+\frac{x^{6}}{360}-\frac{5 x^{5}}{120} \\
& y_{1}(x)=-\frac{\alpha x^{5}}{120}-\frac{x^{6}}{360}+\left(\frac{\alpha}{680}-\frac{\beta}{5040}\right) x^{7}+\frac{1}{3360} x^{8}+\left(\frac{\beta}{36288}+\frac{1}{362880}\right) x^{9}+\cdots \\
& y_{2}(x)=\frac{\alpha}{362880} x^{9}+\cdots, \\
& \ldots,
\end{aligned}
$$

where other $x$ terms and other components $y_{j}$ were also evaluated but not listed. To determine the constants $\alpha$ and $\beta$, we use the boundary conditions $y(1)=0$ and $y^{\prime \prime}(1)=2$ in the the two-stage, three-stage, and fourth-stage approximate solutions, where the following sequences for $\alpha$ and $\beta$

$$
\begin{aligned}
& \alpha=-1.000086673,-1.000006123,-1.000, \\
& \beta=0.0005059937268,0.2465705339 \times 10^{-5}, 0.5906015238 \times 10^{-9}
\end{aligned}
$$

are readily obtained. This shows that $\alpha$ converges to -1 , and $\beta$ converges to 0 . Consequently, we obtain the exact solution

$$
y(x)=x^{2}-x
$$

Example 2. Consider the boundary value problem $[17,20]$

$$
u^{(4)}+f(x) u=0,0<x<1
$$

with the boundary conditions (1.2), where $f(x) \neq k^{4} \pi^{4}, k=1,2, \ldots$. This problem has only the trivial solution $u=0$ as shown in $[17,20]$. Also, the trivial solution obtained $(u=0)$ by the recursion scheme (3.4) to this particular problem is consistent with this result.

Example 3. Consider the boundary value problem

$$
u^{(4)}=k_{1} u-k_{2} u^{\prime \prime}+k_{3} \sin (\pi x), 0<x<1,
$$

with (1.2), where $k_{1}=\frac{\pi^{4}}{4}, k_{2}=\frac{\pi^{2}}{4}, k_{3}=\frac{\pi^{4}}{2}$. Here

$$
f\left(x, u, u^{\prime \prime}\right)=k_{1} u-k_{2} u^{\prime \prime}+k_{3} \sin (\pi x) .
$$

A simple calculation leads to

$$
\left|f\left(x, u, u^{\prime \prime}\right)\right| \leq k_{1}|u|+k_{2}\left|u^{\prime \prime}\right|+k_{3},
$$

where $\frac{k_{1}}{\pi^{4}}+\frac{k_{2}}{\pi^{2}}=\frac{1}{2}<1$. Thus the conditions of Theorem 2 are satisfied and further $f$ satisfies a Lipschitz condition in $u$ and $u^{\prime \prime}$. Then this problem has one solution. 
We accordingly set the following recursion scheme

$$
\left\{\begin{aligned}
u_{0}= & 0, v_{0}=-\left[\int_{0}^{1} \int_{0}^{y} g(t) d t d y\right] x+\int_{0}^{x} \int_{0}^{y} g(t) d t d y \\
u_{n+1}= & -\left[\int_{0}^{1} \int_{0}^{y} v_{n}(t) d t d y\right] x+\int_{0}^{x} \int_{0}^{y} v_{n}(t) d t d y, n \geq 0 \\
v_{n+1}= & -\left[\int_{0}^{1} \int_{0}^{y} f_{1}\left(u_{n}(t), v_{n}(t)\right) d t d y\right] x \\
& +\int_{0}^{x} \int_{0}^{y} f_{1}\left(u_{n}(t), v_{n}(t)\right) d t d y, n \geq 0
\end{aligned}\right.
$$

where $g(x)=k_{3} \sin (\pi x)$ and $f_{1}\left(u_{n}, v_{n}\right)=k_{1} u_{n}-k_{2} v_{n}$.

By computation, we obtain

$$
\left\{\begin{aligned}
u_{0} & =0, v_{0}=-\frac{\pi^{2}}{2} \sin (\pi x), \\
u_{1} & =\frac{1}{2} \sin (\pi x), v_{1}=-\frac{\pi^{2}}{8} \sin (\pi x), \\
u_{2} & =\frac{1}{8} \sin (\pi x), v_{2}=-\frac{5 \pi^{2}}{32} \sin (\pi x), \\
u_{3} & =\frac{5}{32} \sin (\pi x), v_{3}=-\frac{9 \pi^{2}}{128} \sin (\pi x), \\
u_{4} & =\frac{9}{128} \sin (\pi x), v_{4}=-\frac{29 \pi^{2}}{32} \sin (\pi x), \\
u_{5} & =\frac{29}{512} \sin (\pi x), v_{5}=-\frac{65 \pi^{2}}{2048} \sin (\pi x), \\
u_{6} & =\frac{65}{2048} \sin (\pi x), v_{6}=-\frac{181 \pi^{2}}{8192} \sin (\pi x), \\
u_{7} & =\frac{181}{8192} \sin (\pi x), v_{7}=-\frac{441 \pi^{2}}{32768} \sin (\pi x), \\
u_{8} & =\frac{441}{32768} \sin (\pi x), v_{8}=-\frac{1165 \pi^{2}}{131072} \sin (\pi x), \\
& \ldots
\end{aligned}\right.
$$

Consequently, the approximate solution $\phi_{n}(x)=\sum_{k=0}^{n-1} u_{k}$ and the 9th-stage approximation function of $u(x)$ is given as

$$
\begin{aligned}
\phi_{9}(x) & =\sum_{k=0}^{8} u_{k}=u_{0}+u_{1}+u_{2}+\ldots+u_{8} \\
& =\left[\frac{1}{2}+\frac{1}{8}+\frac{5}{32}+\ldots+\frac{181}{8192}+\frac{441}{32768}\right] \sin (\pi x) \\
& =0.976 \sin (\pi x) \approx \sin (\pi x),
\end{aligned}
$$

which is the exact solution to this particular boundary value problem.

Using the standard Adomian decomposition method. In an operator form, we rewrite this equation as

$$
\mathcal{L} u=k_{1} u-k_{2} u^{\prime \prime}+k_{3} \sin (\pi x), 0<x<1
$$

with the given boundary conditions, where $\mathcal{L}$ is a fourth differential operator, where we assume the fourth integral operator $\mathcal{L}^{-1}$ exists. Applying the inverse operator $\mathcal{L}^{-1}$ to both sides of (3.7) gives

$$
u(x)=\alpha x+x^{2}+\frac{\beta x^{3}}{6}+\frac{1}{2} \sin (\pi x)-\frac{\pi x}{2}+\frac{\pi^{3} x^{3}}{12}-\mathcal{L}^{-1}\left(k_{1} u-k_{2} u^{\prime \prime}\right),
$$

where the unknown constants $\alpha=y^{\prime}(0), \beta=y^{\prime \prime \prime}(0)$ will be determined later by using the other boundary conditions. 
Proceeding as in Example 1, we obtain the following solution components

$$
\begin{aligned}
& u_{0}(x)=\alpha x+x^{2}+\frac{\beta x^{3}}{6} x^{3}+\frac{1}{2} \sin (\pi x)-\frac{\pi x}{2}+\frac{\pi^{3} x^{3}}{12} \\
& u_{1}(x)=\left(\frac{\alpha \pi^{4}}{480}-\frac{\beta \pi^{2}}{480}\right) x^{5}+\left(\frac{\beta \pi^{4}}{20160}-\frac{\pi^{7}}{40320}\right) x^{7}+\cdots \\
& u_{2}(x)=\left(\frac{\beta \pi^{4}}{80640}-\frac{\alpha \pi^{6}}{80640}\right) x^{7}+\cdots
\end{aligned}
$$

where other $x$ terms and other components $y_{3}$ and $u_{4}(x)$ were also evaluated but not listed. To determine the constants $\alpha$ and $\beta$, we use the boundary conditions $u(1)=0$ and $u^{\prime \prime}(1)=0$ in the two-stage, three-stage, and fourthstage approximate solution, where the following sequences for $\alpha$ and $\beta$

$$
\begin{aligned}
& \alpha=3.834103992, \quad 3.102954222,3.143078028, \\
& \beta=-36.89714965, \quad-30.69003970, \quad-31.01824125
\end{aligned}
$$

were readily obtained. This means that $\alpha$ converges to $\pi$ and $\beta$ to $-\pi^{3}$. Substituting $\alpha=\pi$ and $\beta=-\pi^{3}$ in the fourth stage approximation, we obtain the series solution

$$
u(x)=\pi x-\frac{\pi^{3}}{3 !} x^{3}+\frac{\pi^{5}}{5 !} x^{5}-\frac{\pi^{7}}{7 !} x^{7}+\cdots,
$$

which is the Taylor series for the exact solution $u(x)=\sin (\pi x)$.

\section{Comparison of the two approximate solutions by the ADM with the Green function approach for two similar equations}

In this section, we compare two approximate solutions obtained by the Adomian decomposition method (ADM) with the Green function approach for two similar equations. Green's functions for BVP (differential and discrete) were investigated in $[12,13,15]$ (see a survey [14], too). Consider the nonlinear boundary value problem [4]

$$
u^{(4)}=\lambda f(x, u), 0<x<1, \lambda>0
$$

subject to the set of boundary conditions (1.2).

This problem can be converted into the following system

$$
\left\{\begin{array}{l}
u^{\prime \prime}=v, u(0)=u(1)=0, \\
v^{\prime \prime}=\lambda f(x, u), v(0)=v(1)=0 .
\end{array}\right.
$$

Then problem (4.2) is equivalent to the system of coupled integral equations

$$
\left\{\begin{array}{l}
u=\int_{0}^{1} G(x, y) v(y) d y \\
v=\lambda \int_{0}^{1} G(x, y) f(y, u(y)) d y
\end{array}\right.
$$

where $G(x, y)$ is the Green function defined as

$$
G(x, y)= \begin{cases}x(y-1), & 0 \leq x \leq y \leq 1 \\ y(1-x), & 0 \leq y \leq x \leq 1\end{cases}
$$


It follows that

$$
u(x)=\lambda \int_{0}^{1} K(x, y) f(y, u(y)) d y
$$

which is expressed in the canonical form, where $K(x, y)=\int_{0}^{1} G(x, t) G(t, y) d t$. The same procedure can be adopted to resolve this nonlinear integral equation. We have

Theorem 3. Let $\Phi_{n}(x)=\sum_{i=0}^{n-1} u_{i}(x)$ and $\Psi_{n}(x)=\sum_{i=0}^{n-1} u_{i}(x)$ be two nthstage approximation functions of $u(x)$ by the ADM for the nonlinear boundary value problems equation (4.1) and

$$
u^{(4)}=\lambda g(x, u), 0<x<1
$$

with (1.2), respectively such that $f, g$ satisfy the Lipschitz condition in $u$ and $|K(x, y)| \leq M, 0 \leq x \leq 1,0 \leq y \leq 1$. If

$$
\max _{0 \leq x \leq 1}\left|f\left(x, \Psi_{n}(x)\right)-g\left(x, \Psi_{n}(x)\right)\right| \leq \varepsilon, \varepsilon>0,
$$

then

$$
\left\|\Phi_{n}-\Psi_{n}\right\|_{C[0,1]} \leq \frac{\lambda M \varepsilon}{1-\lambda k M}
$$

where $0<k<\frac{1}{\lambda M}$ is a Lipschitz constant.

Proof. We have

$$
\begin{aligned}
& \Phi_{n}(x)=\lambda \int_{0}^{1} K(x, y) f\left(y, \Phi_{n}(y)\right) d y, \quad n \geq 1 \\
& \Psi_{n}(x)=\lambda \int_{0}^{1} K(x, y) g\left(y, \Psi_{n}(y)\right) d y, \quad n \geq 1
\end{aligned}
$$

with $\Phi_{n}(0)=\Psi_{n}(0)=0$. It follows that

$$
\max _{0 \leq x \leq 1}\left|\Phi_{n}(x)-\Psi_{n}(x)\right| \leq \lambda M \max _{0 \leq x \leq 1}\left|f\left(x, \Phi_{n}(x)\right)-g\left(x, \Psi_{n}(x)\right)\right| .
$$

Since

$$
\left|f\left(x, \Phi_{n}\right)-g\left(x, \Psi_{n}\right)\right|=\left|f\left(x, \Phi_{n}\right)-f\left(x, \Psi_{n}\right)+f\left(x, \Psi_{n}(x)\right)-g\left(x, \Psi_{n}\right)\right| .
$$

Thus

$$
\max _{0 \leq x \leq 1}\left|\Phi_{n}(x)-\Psi_{n}(x)\right| \leq \lambda M\left[k \max _{0 \leq x \leq 1}\left|\Phi_{n}(x)-\Psi_{n}(x)\right|+\varepsilon\right] .
$$

Consequently,

$$
\max _{0 \leq x \leq 1}\left|\Phi_{n}(x)-\Psi_{n}(x)\right| \leq \frac{\lambda M \varepsilon}{1-\lambda k M},
$$

and we obtain (4.3). 
Theorem 4. Let $\Phi_{n}(x)$ and $\Psi_{n}(x)$ be two nth-stage $\varepsilon_{i}-$ approximation functions of $u(x)$ by the ADM for the nonlinear boundary value problems equation (4.1) with (1.2), that is there exist $\varepsilon_{i}, i=1,2$ such that

$$
\begin{aligned}
& \left|\Phi_{n}(x)-\lambda \int_{0}^{1} K(x, y) f\left(y, \Phi_{n}(y)\right) d y\right| \leq \varepsilon_{1} \\
& \left|\Psi_{n}(x)-\lambda \int_{0}^{1} K(x, y) f\left(y, \Psi_{n}(y)\right) d y\right| \leq \varepsilon_{2} .
\end{aligned}
$$

Under the same hypothesis on $f$ and $K(x, y)$ of Theorem 3, we have

$$
\left\|\Phi_{n}-\Psi_{n}\right\|_{C[0,1]} \leq \frac{\varepsilon_{1}+\varepsilon_{2}}{1-\lambda k M}
$$

Proof. We have

$$
\begin{aligned}
& \left|\Phi_{n}(x)-\Psi_{n}(x)\right| \leq \mid \Phi_{n}(x)-\lambda \int_{0}^{1} K(x, y) f\left(y, \Phi_{n}(y)\right) d y+\lambda \int_{0}^{1} K(x, y) \\
& \quad \times f\left(y, \Psi_{n}(y)\right) d y-\Psi_{n}(x)|+\lambda| \int_{0}^{1} K(x, y)\left[f\left(y, \Phi_{n}(y)\right)-f\left(y, \Psi_{n}(y)\right)\right] d y \mid .
\end{aligned}
$$

So that

$$
\left|\Phi_{n}(x)-\Psi_{n}(x)\right| \leq \varepsilon_{1}+\varepsilon_{2}+\lambda k M \max _{0 \leq x \leq 1}\left|\Phi_{n}(x)-\Psi_{n}(x)\right| .
$$

Consequently

$$
\max _{0 \leq x \leq 1}\left|\Phi_{n}(x)-\Psi_{n}(x)\right| \leq \frac{\varepsilon_{1}+\varepsilon_{2}}{1-\lambda k M}
$$

and we obtain (4.4).

\section{References}

[1] G. Adomian. Modification of the decomposition approach to the heat equation. Journal of Mathematical Analysis and Applications, 124(1):290-291, 1987. http://dx.doi.org/10.1016/0022-247X(87)90040-0.

[2] G. Adomian. Nonlinear Stochastic Systems theory and applications to physics. Kluwer Academic Publishers Group, Dordrecht, 1989.

[3] G. Adomian. Solving frontier problems of physics: the decomposition method. Kluwer Academic Publishers, Dordrecht, 1994. http://dx.doi.org/10.1007/97894-015-8289-6.

[4] Z. Bai and H. Wang. On positive solutions of some nonlinear fourth-order beam equations. Journal of Mathematical Analysis and Applications, 270(2):357-368, 2002. http://dx.doi.org/10.1016/S0022-247X(02)00071-9. 
[5] L. Bougoffa, R. Rach, A.-M. Wazwaz and J.-S. Duan. On the Adomian decomposition method for solving the Stefan problem. International Journal of Numerical Methods for Heat \& Fluid Flow, 25(4):912-928, 2015. http://dx.doi.org/10.1108/HFF-05-2014-0159.

[6] P. Dràbek and G. Holubovà. Positive and negative solutions of onedimensional beam equation. Applied Mathematics Letters, 51:1-7, 2016. http://dx.doi.org/10.1016/j.aml.2015.06.019.

[7] J.-S. Duan and R. Rach. A new modification of the Adomian decomposition method for solving boundary value problems for higher order nonlinear differential equations. Applied Mathematics and Computation, 218(8):4090-4118, 2011. http://dx.doi.org/10.1016/j.amc.2011.09.037.

[8] J.-S. Duan, R. Rach and A.-M. Wazwaz. Solution of the model of beam-type micro- and nano-scale electrostatic actuators by a new modified Adomian decomposition method for nonlinear boundary value problems. International Journal of Non-Linear Mechanics, 49:159-169, 2013. http://dx.doi.org/10.1016/j.ijnonlinmec.2012.10.003.

[9] J.-S. Duan, R. Rach and A.-M. Wazwaz. A reliable algorithm for positive solutions of nonlinear boundary value problems by the multistage Adomian decomposition method. Open Engineering, 5(1):59-74, 2014. http://dx.doi.org/10.1515/eng-2015-0007.

[10] J.-S. Duan, R. Rach, A.-M. Wazwaz, T. Chaolu and Z. Wang. A new modified Adomian decomposition method and its multistage form for solving nonlinear boundary value problems with Robin boundary conditions. Applied Mathematical Modelling, 37(20-21):8687-8708, 2013. http://dx.doi.org/10.1016/j.apm.2013.02.002.

[11] J. R. Graef, J. Henderson and B. Yang. Positive solutions to a fourth order three point boundary value problem. Discrete and Continuous Dynamical Systems, 2009:269-275, 2009. http://dx.doi.org/10.3934/proc.2009.2009.269.

[12] G. Paukštaitè and A. Štikonas. Ordinary and generalized Green's functions for the second order discrete nonlocal problems. Boundary Value Problems, 2015(1):207, 2015. http://dx.doi.org/10.1186/s13661-015-0474-6.

[13] S. Roman. Linear differential equation with additional conditions and formulae for Green's function. Mathematical Modelling and Analysis, 16(3):401-417, 2011. http://dx.doi.org/10.3846/13926292.2011.602125.

[14] A. Štikonas. A survey on stationary problems, Green's functions and spectrum of Sturm-Liouville problem with nonlocal boundary conditions. Nonlinear Analysis: Modelling and Control, 19(3):301-334, 2014. http://dx.doi.org/10.15388/NA.2014.3.1.

[15] A. Štikonas and S. Roman. Stationary problems with two additional conditions and formulae for Green's functions. Numerical Functional Analysis and Optimization, 30(9-10):1125-1144, 2009. http://dx.doi.org/10.1080/01630560903420932.

[16] S. Timoshenko and S. Woinowsky-Krieger. Theory of plates and shells. McGrawHill, New York, 1959.

[17] R.A. Usmani. A uniqueness theorem for a boundary value problem. Proceedings of the American Mathematical Society, 77(3):329-335, 1979. http://dx.doi.org/10.1090/S0002-9939-1979-0545591-4. 
[18] A.-M. Wazwaz. Partial Differential Equations and Solitary Waves Theory. Nonlinear Physical Science. Springer Berlin Heidelberg, 2009. http://dx.doi.org/10.1007/978-3-642-00251-9.

[19] A.-M. Wazwaz. Linear and Nonlinear Integral Equations: Methods and Applications. Springer-Verlag Berlin Heidelberg, 2011. http://dx.doi.org/10.1007/9783-642-21449-3.

[20] Y.S. Yang. Fourth-order two-point boundary value problems. Proceedings of the American Mathematical Society, 104(1):175-180, 1988. http://dx.doi.org/10.1090/S0002-9939-1988-0958062-3. 